\title{
SISTEMAS DE ALERTA TEMPRANA CON ENFOQUE PARTICIPATIVO: UN DESAFÍO PARA LA GESTIÓN DEL RIESGO EN COLOMBIA
}

\author{
Juan-David López-García \\ Yesid Carvajal-Escobar (iD 2 \\ Angélica-María Enciso-Arango (iD ${ }^{3}$
}

Recibido el 16 de septiembre de 2015, aprobado el 5 de mayo de 2016 y actualizado el

20 de diciembre de 2016

DOI: 10.17151/luaz.2017.44.14

\section{RESUMEN}

La mayor parte de la población colombiana está en condiciones de riesgo debido a factores hidroclimatológicos, fuertemente influenciados por la variabilidad (VC) y el cambio climático (CC). A esto se suma el aumento de la vulnerabilidad por la inadecuada planificación y ocupación del territorio, propia de países en desarrollo. En este sentido, los sistemas de alerta temprana (SAT) facilitan los procesos de adaptación y mitigación de impactos, por lo que se constituyen en uno de los ejes transversales de la gestión del riesgo. En este trabajo se presenta una reflexión de diversos enfoques y experiencias nacionales e internacionales en SAT. A partir de lo anterior, se identificó que muchos SAT no llegan a ser implementados; y una vez en funcionamiento, existe desequilibrio entre sus componentes. Por otro lado, no se ajustan a las necesidades de los territorios como consecuencia de la escasa participación comunitaria, tanto en la etapa de diseño como en la de operación. Por ello, se considera que para obtener resultados eficaces, que promuevan la construcción de resiliencia, son necesarios SAT con enfoque participativo, que fortalezcan las capacidades de las comunidades para enfrentar las condiciones de riesgo de su entorno.

Palabras clave: Variabilidad climática, cambio climático, vulnerabilidad, sistema de alerta temprana, gestión del riesgo.

\section{EARLY WARNING SYSTEMS WITH A PARTICIPATIVE APPROACH: A CHALLENGE FOR RISK MANAGEMENT IN COLOMBIA}

\section{ABSTRACT}

Most of the Colombian population is facing risks due to hydroclimatological factors, strongly influenced by the climate variability (CV) and climate change (CC). Likewise, vulnerability is continuously increasing because of inadequate land planning and occupation, common in developing countries. Therefore, early warning systems (EWS) facilitate the processes of adaptation and mitigation of impacts, which is why they constitute one of the transverse axes in risk management. This study aimed to review approaches to and field experiences with EWS throughout the world, including Colombia. We identified that many EWS are unimplemented; and once in operation, there exists an imbalance among components. On the other hand, some EWS fail to meet the territory needs as a result of poor community participation, both at design and operation stages. Hence, a participative approach in EWS to tackling risks and building up the capacities of the communities should be promoted for obtaining effective results.

Key words: Climate variability, climate change, vulnerability, early warning systems, risk management. 


\section{INTRODUCCIÓN}

El cambio climático (CC) y en especial la variabilidad climática (VC) ocasionan frecuentes anomalías en el ciclo hidrológico, que impactan negativamente los ecosistemas y la sociedad (Sánchez et al, 2011; Vincent, 2007; Brown y Funk, 2008). Según el Grupo Intergubernamental de Expertos sobre el Cambio Climático (IPCC por sus siglas en inglés), en América, Asia y el norte de Europa existen tendencias al incremento de la precipitación, aumentando la probabilidad de ocurrencia e intensidad de eventos extremos y desastres como deslizamientos e inundaciones (IPCC, 2012), que dadas las crecientes condiciones de vulnerabilidad y pobreza en el mundo, podrían aumentar en un 50\% el número de personas afectadas para 2015, alcanzando 375 millones de damnificados (Petrone y Petri, 2011). Sin embargo, las amenazas naturales por sí solas no ocasionan desastres; estos se generan por la combinación de factores como una población expuesta, vulnerable y poco preparada, enfrentada a dichas amenazas (ONU y EIRD, 2008). Es por esto que a pesar de que los efectos del CC afectarán a escala mundial, los países más pobres serán los más damnificados, debido a la vulnerabilidad de su población; por la localización en zonas de alto riesgo; y por la falta de políticas y tecnologías adecuadas para prevenir, controlar y mitigar estos eventos y sus impactos.

Colombia es un país altamente vulnerable a los efectos del CC y la VC, por la recurrencia de eventos climáticos extremos, siendo El Niño Oscilación del Sur (ENOS), en sus fases, cálida (EI Niño) y fría (La Niña); el modo dominante de VC y el fenómeno natural de mayor impacto socioeconómico en Latinoamérica, por su potencial para desencadenar sequías, inundaciones, olas de frío o de calor y tormentas, entre otros (Carvajal et al., 2007; Bedoya et al., 2010; García et al, 2012). Un ejemplo de pérdidas económicas por eventos climáticos es el fenómeno "La Niña" de 2010-2011, que dejó cerca de 5,2 millones de personas afectadas y pérdidas económicas estimadas en $\$ 8,6$ billones, reflejando la complejidad de las condiciones de riesgo existentes en el país y los vacíos y deficiencias en la capacidad de gestión del riesgo (Campos et al, 2012; Sedano et al, 2013).

Según el informe Stern (2007), actuar hoy frente al CC resulta menos costoso que afrontar las pérdidas que generarían sus impactos futuros sobre la economía. Entre las estrategias para enfrentar el CC, la gestión del riesgo representa un elemento clave, ya que trata de suministrar información a la comunidad sobre los distintos fenómenos hidrometeorológicos, con el fin de reducir sus impactos. De las diferentes acciones que se enmarcan en este concepto, los sistemas de alertas tempranas (SAT) son una de las principales estrategias de adaptación frente al CC, que procuran evitar pérdida de vidas y disminuir los impactos económicos, sociales y ambientales, especialmente en las poblaciones más vulnerables. La eficacia de estos sistemas radica en la participación activa de las comunidades, siendo la educación, la base para tomar conciencia de los riesgos a los que se enfrentan.

La implementación de los SAT, como estrategia para reducir los efectos de la VC y el CC, cobra cada vez más relevancia, y aunque existe abundante información, ésta en muchos casos se encuentra dispersa, aislada, incompleta o no es asequible. En Colombia existen experiencias interesantes de implementación de SAT locales, sin embargo, la mayoría de los casos no están sistematizados o no se han recopilado en un conjunto de estrategias que recomienden las lecciones aprendidas de los mismos. Este trabajo, analiza y evidencia la motivación y situación actual de los SAT en 
Colombia, para diagnosticar su estado y compararlo con tendencias latinoamericanas y mundiales, con el fin de ofrecer lineamientos y recomendaciones para un eficaz y mejor uso.

\section{METODOLOGÍA}

Se revisaron experiencias cuyo criterio de búsqueda incluye artículos científicos, informes y documentos institucionales, libros y archivos periodísticos. Se realizó una búsqueda sistematizada de información para aumentar la probabilidad de hallar la cantidad adecuada de documentos pertinentes y relevantes sobre el tema; identificando, analizando y sintetizando información sobre diferentes conceptos, enfoques y experiencias exitosas en SAT, tanto del ámbito nacional como internacional. Así mismo, se identificaron y analizaron los principales desafíos que enfrentan estas herramientas en el futuro para luego ofrecer recomendaciones aplicables al contexto colombiano, con lineamientos que permitan mejorar las deficiencias que dichos sistemas presentan para el logro de un funcionamiento más eficaz.

\section{MARCO DE CONTEXTO}

\section{- Generalidades de la hidroclimatología colombiana}

La hidroclimatología de Colombia está principalmente determinada por: (1) su ubicación en la franja ecuatorial y en el trópico americano, bajo la influencia de los vientos alisios y la oscilación meridional de la zona de convergencia intertropical (ZCIT), que determina la distribución espacio-temporal de la precipitación y de otras variables hidroclimatológicas en el país; (2) su localización al noroccidente de Suramérica, que permite la influencia de procesos que ocurren en los océanos Pacífico y Atlántico, como importantes fuentes de humedad atmosférica que penetran hacia el interior del país; (3) la influencia de la circulación atmosférica de la cuenca Amazónica; (4) su formación fisiográfica que incluye la cordillera de los Andes, como barrera orográfica, con valles interandinos y vertientes de cordillera, que inducen la formación de climas locales y regionales complejos; (5) la variabilidad de los procesos de la hidrología superficial, tales como los contrastes en humedad de suelo y evapotranspiración, fuertemente influenciados por la vegetación, el tipo de suelos y la circulación de vientos locales; (6) y la interacción océano-tierra-atmósfera sobre la franja más al este del Océano Pacífico tropical, realzada por la dinámica del "chorro del Chocó", una corriente de vientos húmedos de bajo nivel, que penetra al territorio colombiano (Poveda, 2004; Poveda y Salazar, 2004; Puertas y Carvajal, 2008; García et al., 2012; Carvajal et al, 2014).

La localización del país en la franja ecuatorial hace especialmente difícil separar los efectos del CC y la VC, dado que la última ejerce una fuerte influencia en las condiciones normales del clima, y sus efectos no se perciben de manera homogénea en el territorio nacional (García et al, 2012). En Colombia, el efecto de la VC natural suscita enormes riesgos a los sectores sociales y económicos, debido a la intensidad de los eventos que presentan y que están influenciados principalmente por el fenómeno ENOS (Montealegre y Pabón, 2000). La influencia del ENOS y su impacto, es diferente para cada región y depende de factores como su proximidad al océano Pacífico, siendo el occidente y centro del país, las regiones donde más se evidencian sus efectos. 


\section{- Efectos del ENOS en el contexto nacional}

En Colombia, durante la fase fría del ENOS (La Niña), la persistencia de las lluvias satura de humedad el suelo, originando crecientes súbitas, avalanchas, deslizamientos e inundaciones. Caso contrario ocurre durante su fase cálida (EI Niño); la ausencia de precipitaciones genera sequías extremas, que ocasionan déficit de suministro de agua potable, producción agrícola y energía eléctrica e incendios forestales, entre otros (Mantilla et al., 2009; Puertas et al., 2011; Carvajal, 2011). Los impactos que generan ambas fases, dependen y están relacionados con el grado de vulnerabilidad de las diferentes regiones y de los sectores de la actividad nacional.

Durante La Niña se incrementan las inundaciones, siendo los desastres que más daños provocan en el mundo, con mayores efectos negativos en las poblaciones de los países más pobres, seguidas de los movimientos en masa, ocasionados por intervenciones antrópicas o por procesos naturales (Petrone y Petri, 2011; Aristizábal et al, 2011). En Colombia, el $28 \%$ del territorio presenta un alto potencial de inundaciones y el $8 \%$ amenaza alta por movimientos en masa (Campos et al., 2012). Estos eventos han demostrado históricamente su capacidad destructora, ocasionando cuantiosas pérdidas humanas y económicas, especialmente en zonas tropicales y terrenos montañosos, que por sus características fisiográficas y socioeconómicas presentan una alta susceptibilidad a desarrollar este tipo de procesos (Schuster, 1996). Los últimos 40 años han estado marcados por más de 28 mil eventos desastrosos, cuyas pérdidas rondan los US $\$ 7.100$ millones. La susceptibilidad a fenómenos hidrometeorológicos ha crecido en aproximadamente un $330 \%$, debido a procesos inadecuados de ordenamiento territorial, degradación ambiental y factores asociados con la VC, entre otros (Campos et al., 2012). Las cifras demuestran la necesidad de incorporar nuevas herramientas para la gestión del riesgo en el país, como estrategia para reducir los efectos de la VC y el CC, con un enfoque diferente y orientado a la integración y participación de las comunidades; así mismo, que consideren procesos integrales, que permitan reducir el nivel de afectación de estos fenómenos. De esta forma, cobran relevancia herramientas como los SAT, que permitirían mejorar la capacidad de adaptación a eventos extremos en los próximos años.

\section{- La gestión del riesgo en Colombia}

En Colombia el $82 \%$ de la población está bajo condiciones de riesgo debido factores geológicos, geomorfológicos, hidroclimáticos, y al creciente aumento de la vulnerabilidad, como resultado de la inadecuada planificación y ocupación del territorio. Así mismo, se tienen asentamientos con infraestructura deficitaria y altos índices de pobreza. La vulnerabilidad es el resultado no sólo de la ocurrencia de fenómenos peligrosos, si no de la forma en que las sociedades se han desarrollado, se organizan, se preparan para enfrentarlos y se recuperan de ellos (Landa et al., 2008; Campos et al., 2012; Fernández y Sabas, 2012).

El gobierno nacional ha venido modificando la estructura normativa para hacer frente a los desastres, con el fin de reducir o evitar impactos de gran magnitud. Se ha pasado así del concepto de "atención de desastres", con la creación del Sistema Nacional para la Prevención y Atención de Desastres en el año 1989, a la "gestión del riesgo", con la sanción de la Ley 1523 de 2012, por la cual se establece el Sistema Nacional de Gestión del Riesgo de Desastre, en cabeza de la UNGRD (Figura 1) (UNGRD, 2012; Colombia Humanitaria, 2012). 


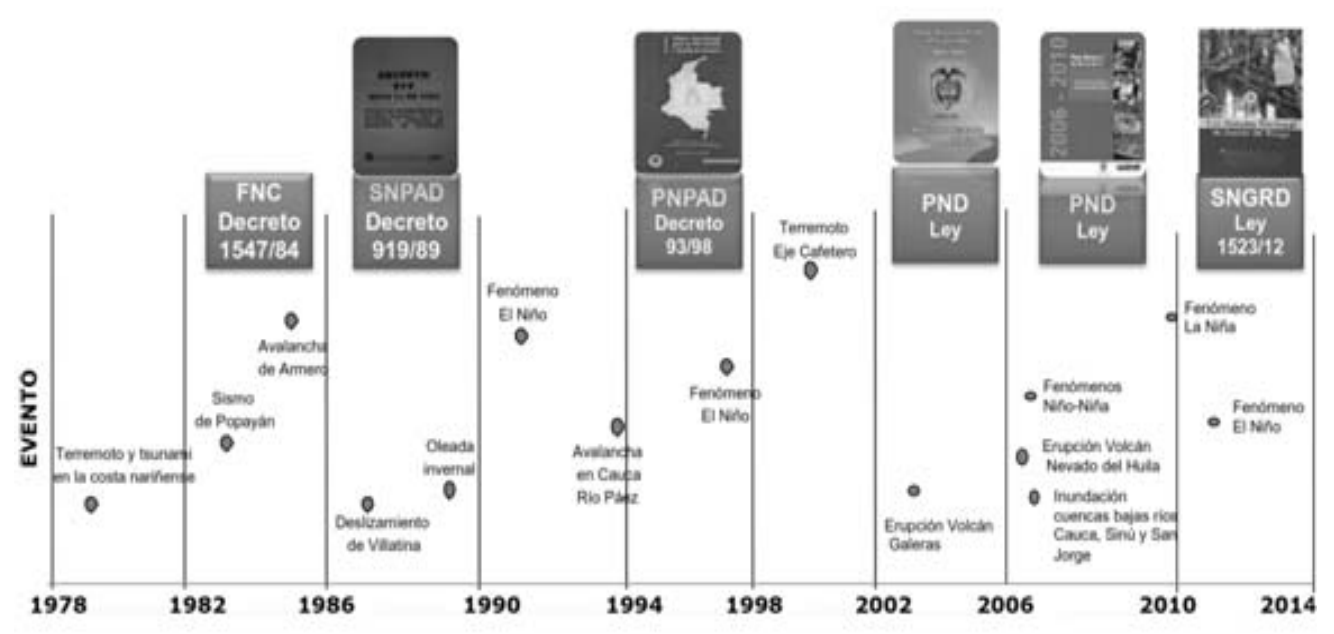

Fuente: UNGRD, S.f.

Figura 1. Evolución de la gestión del riesgo en Colombia en función de los

\section{eventos desastrosos.}

Como consecuencia de los cambios de enfoque en las políticas nacionales, el país ha adquirido compromisos en el marco de acuerdos internacionales, como el Marco de Acción de Hyogo para 2005-2015, "Aumento de la resiliencia de las naciones y las comunidades ante los desastres", aprobado durante la Segunda Conferencia Mundial sobre Reducción de Desastres llevado a cabo en Hyogo-Japón. El marco cuenta con cinco prioridades de acción: 1) velar porque la reducción del riesgo de desastres constituya una prioridad nacional y local; 2) identificar, evaluar y seguir de cerca el riesgo de desastres y potenciar la alerta temprana; 3) utilizar el conocimiento, la innovación y la educación para establecer una cultura de seguridad y de resiliencia; 4) reducir los factores subyacentes del riesgo; y 5) fortalecer la preparación ante los desastres para lograr una respuesta eficaz a todo nivel. Con base en estas prioridades, los SAT toman gran relevancia en la gestión del riesgo nacional.

\section{- Conceptos básicos y componentes de un sistema de alerta temprana (SAT)}

Los SAT son definidos como el conjunto de dispositivos y capacidades necesarios para generar y difundir una alerta oportuna, que avise sobre la posibilidad de ocurrencia de un evento de origen natural o antrópico, que puede desencadenar un desastre, con el fin de evitar o mitigar sus impactos (Ocharan, 2007; OEA, 2010; Domínguez y Lozano, 2014). De acuerdo con la United Nations International Strategy for Disaster Reduction- (UNISDR, 2009), un SAT comprende cuatro elementos fundamentales (Figura 2): 1) conocimiento del riesgo; 2) el monitoreo, análisis y pronóstico de la amenaza; 3) comunicación o difusión de las alertas y los avisos; y 4) capacidades locales para responder frente a la alerta recibida. 


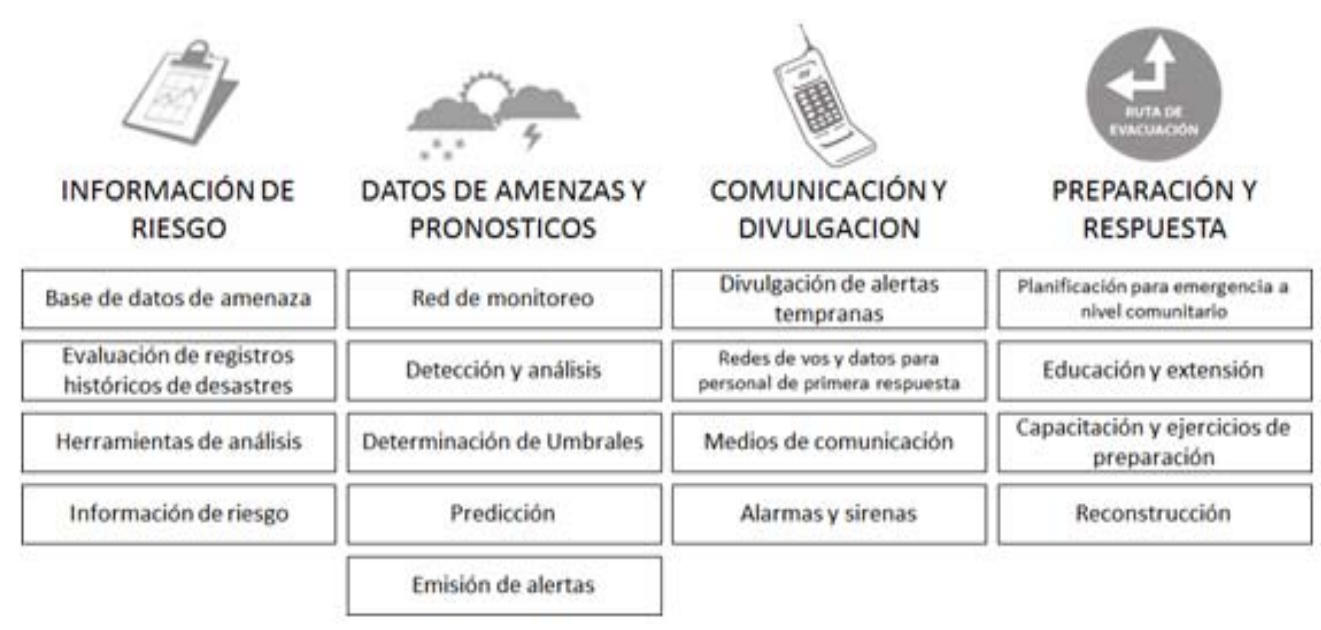

Fuente: Adaptado de NOAA, 2012.

Figura 2.Componentes de un SAT.

Los SAT que involucran a la población y que se ajustan a las realidades territoriales, tanto en la fase de diseño como de operación, representan un eje transversal en la gestión del riesgo y deben estar presentes en varios de sus procesos, ya que integran aspectos misionales e incorporan todos sus componentes: prospectivo, correctivo y reactivo (Figura 3), específicamente los de tipo reactivo; partiendo del análisis de la amenaza, el establecimiento de sistemas de monitoreo para la alerta y la preparación de la población para actuar frente al riesgo (UNESCO, 2012).

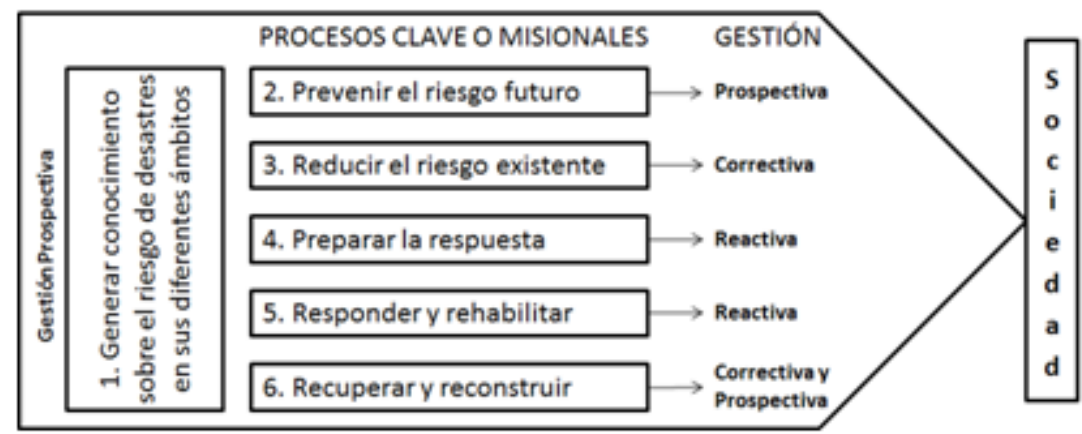

Fuente: Adaptado de, Narváez et al., 2009; Lozano, 2011.

Figura 3. Mapa de procesos y componentes de la gestión

del riesgo de desastres

\section{ELEMENTOS DE LOS SAT Y SU FUNCIONAMIENTO EN COLOMBIA}

\section{- Conocimiento del riesgo}

La base para la construcción de un SAT, es la información que se pueda obtener sobre los eventos que han desencadenado desastres en un territorio, y su efectividad depende de la calidad y cantidad de la misma. En este caso, los inventarios de desastre son una herramienta fundamental que permite identificar las comunidades 
que se encuentran en riesgo y las amenazas de su entorno, estableciendo la relación entre el desastre y el evento que lo desencadena.

Actualmente existen herramientas para construir inventarios de desastres que facilitan el manejo de la información; desde algunas relativamente sencillas como Excel, hastasoftware especializado como DesInventar; desarrollado por La Red de Estudios Sociales en Prevención de Desastres en América Latina, y disponible en la web (www.desinventar.org). Aunque estas herramientas tienen gran potencial, requieren amplia información y de calidad para su óptimo aprovechamiento; lo cual es una de las principales limitantes en Colombia, ya que en general la medición de las variables asociadas a amenazas hidrometeorológicas, presentan series muy cortas y vacíos de información, principalmente en zonas montañosas, donde se concentra gran parte de la población más vulnerable del territorio nacional.

No obstante, es destacable el caso del Valle de Aburrá, donde se ha venido gestando un buen manejo en la generación, sistematización de información y cobertura; gracias a ello, es posible encontrar trabajos que evidencian la buena disponibilidad de información con la que cuenta la zona, como los realizados por: Aristizábal et al. (2011), Moreno et al. (2006), Echeverry y Valencia (2004), entre otros.

\section{- Monitoreo y pronóstico}

El desarrollo de servicios de monitoreo y de pronósticos hidrológicos en tiempo real, aplicados a los SAT, es una tendencia mundial que no ha sido ajena a Colombia; donde además, se han integrado al uso de los ordenadores y medios de comunicación, como parte de la estrategia del país para la reducción del riesgo en desastres de origen hidrometeorológico (Domínguez et al., 2010). Prueba de ello es el Convenio Interadministrativo entre el Fondo Adaptación y el IDEAM (Fondo Adaptación, 2012), con el cual se pretende adquirir nuevas estaciones de monitoreo y repotenciar las exitentes, además de implementar un sistema para la integración de la información generada por los equipos de observacion. Sin embargo, es importante no exceder la capacidad tecnológica del país, apuntando hacia un equilibrio acorde con la realidad y el contexto nacional.

Es claro que los SAT no deben ser tan simples que no cumplan con su cometido, ni tan complejos que no se puedan mantener sin ayuda externa (Ocharan, 2007); factor que incrementa considerablemente los costos de cualquier proyecto, volviéndolo insostenible en el tiempo; ya que además de crear dependencia tecnológica de otros países, no necesariamente aporta a la construcción de resiliencia social y reducción de la vulnerabilidad estructural. Un caso exitoso en Colombia es el SAT de Medellín y el Valle de Aburrá (SIATA), que actualmente utiliza tecnología desarrollada en la región, lo cual se traduce en reducción de costos de instalación, operación y de los tiempos de respuesta para dar solución a cualquier falla en el sistema (Campos et al, 2012).

Aunque en el país se vienen realizando mejoras en el funcionamiento de los SAT, aún persiste la tendencia de concentrar grandes inversiones en el fortalecimiento de componentes tecnológicos, porque estos ofrecen sensación de control y anticipo ante los fenómenos de la naturaleza. Trabajos como los de Acosta (2013), Campo y Zafra (2013), la Agencia Suiza para el Desarrollo y COSUDE (2010) y Corpoguajira (2011), lo demuestran. No obstante, aunque la inversión tecnología es importante, porque permite identificar y pronosticar con precisión la ocurrencia de fenómenos que representan riesgo; los demás elementos del SAT también lo son, es decir, cada uno debe funcionar adecuadamente y en forma articulada; de lo contrario, el sistema de alerta no dará el resultado esperado. Los SAT, además de alertar con suficiente antelación, deben apoyar a la comunidad en la toma de decisiones, en la difusión de la 
alerta y en la ejecución de acciones de respuesta eficaces. Un ejemplo de lo que puede suceder cuando el fortalecimiento de los SAT es desbalanceado, es el caso de Sri Lanka, un país pobre y en conflicto que en 2005 adquirió un sistema de alerta de tsunamis con tecnología japonesa, que hasta el 2007 no había sido puesto en funcionamiento, debido a que no consideró la capacidad de gestión del país para fortalecer los componentes restantes del SAT; es decir, los mecanismos de difusión de las alertas, la preparación y elaboración de planes de respuesta con la población, etc. (Ocharan, 2007).

Adicionalmente, para la implementación de un SAT es importante tener en cuenta los saberes ancestrales tradicionales, que además de ofrecer la posibilidad de complementar las tecnologías modernas, pueden facilitar el proceso de asimilación de las comunidades, dando legitimidad a estos procesos. En Latinoamérica hay numerosos casos que integran los SAT con los saberes ancestrales: el SAT del río Piedras en el macizo colombiano, donde plantean el uso de señales o bioindicadores de conocimiento ancestral; el Plan Comunal de Gestión de Riesgo Agrícola, GRAC, en Bolivia (Quispe y Aguilar, 2009) y el uso de tecnologías económicas con enfoque comunitario en los SAT contra inundaciones en Perú y Centroamerica, (Ferradas y Vilela, 2009; Villagrán et al., 2003) son algunos ejemplos. La participación activa de las comunidades en estos sistemas ha permitido desarrollar respuestas multiobjetivo, considerando que cada grupo social cuenta con vulnerabilidades distintas en función de su cultura, género y otras características.

\section{- Comunicación y divulgación de alertas}

De acuerdo con los compromisos adquiridos por Colombia en el $\mathrm{MAH}^{4}$, se han logrado avances en el fortalecimiento de los sistemas de comunicación para difusión de las alertas tempranas en tiempo real, a través de la actualización, ampliación y optimización de las redes, coberturas y canales de comunicación. Una de las estrategias de comunicación con mayor potencial, son los portales en la web (Tabla 1), desarrollados para orientar, alertar y generar conciencia ciudadana en torno a la gestión del riesgo. Sin embargo, existen algunos casos como La Guajira, Norte de Santander y el río Combeima, donde los SAT se encuentran desactualizados y los procesos de pronóstico y difusión de alertas no son claros; situación por la cual autores como Domínguez y Lozano (2014) cuestionan el cumpliendo de su objetivo.

Estas plataformas enfrentan otra dificultad relacionada con la cobertura. La brecha digital entre países desarrollados y en desarrollo, como Colombia, sigue siendo muy grande, y el acceso a servicios como telefonía móvil e internet, más allá de las ciudades principales, aún es muy limitado; según el Plan Nacional de Desarrollo 2014 - 2018, se estima que sólo el $51,7 \%$ del total de la población tiene internet y sólo el $6,8 \%$ de los hogares en la zona rural tiene acceso al mismo. Por esta razón, se deben identificar canales de difusión más adecuados en cada caso, con el fin de suministrar información clara, útil, oportuna y accesible a todas las personas; principalmente a las que se encuentran en mayor riesgo. En algunas situaciones, será posible hacer uso de tecnologías como celulares, televisión, radio o plataformas web; en otras, por ejemplo, para las comunidades más apartadas, se requerirán medios más sencillos como banderas o sirenas. 
Tabla 1. Sistemas de alerta temprana implementados en el territorio nacional.

\begin{tabular}{|c|c|c|c|c|}
\hline \multirow{2}{*}{ Ubicación } & \multicolumn{3}{|c|}{ Tipo de SAT } & \multirow{2}{*}{ Accesibilidad } \\
\hline & Nivel & Amenaza & Enfoque & \\
\hline $\begin{array}{l}\text { Norte de } \\
\text { Santander }\end{array}$ & Departamental & Multi-amenaza & Centralizado & http://www.satnortedesantander.org/ \\
\hline La Guajira & Departamental & $\begin{array}{l}\text { Inundaciones y } \\
\text { Deslizamientos }\end{array}$ & Centralizado & $\begin{array}{c}\text { http://corpoguajira.gov.co/wp/program } \\
\text { as-y-proyectos/sistema-de-alerta- } \\
\text { temprana/ }\end{array}$ \\
\hline Tolima & Departamental & Inundaciones & Centralizado & http://www.cdgrdtolima.gov.co/ \\
\hline $\begin{array}{l}\text { Valle de } \\
\text { Aburrá }\end{array}$ & Municipal & Multi-amenaza & Centralizado & $\begin{array}{l}\text { http://Www.siata.gov.co/newpage/inde } \\
\text { x.php }\end{array}$ \\
\hline Barranquilla & Municipal & Inundaciones & Centralizado & http://www.arroyosdebarranquilla.co/ \\
\hline Manizales & Municipal & Deslizamientos & Centralizado & $\begin{array}{c}\text { http://idea.manizales.unal.edu.co/inde } \\
\text { x.php/estado-tiempo-caldas } \\
\end{array}$ \\
\hline IDEAM & Nacional & Multi-amenaza & Centralizado & $\begin{array}{c}\text { http://pronosticos.ideam.gov.co/jsp/74 } \\
6\end{array}$ \\
\hline Bogotá & Municipal & Multi-amenaza & Centralizado & http://WwW.sire.gov.col \\
\hline SNGRD & Nacional & Multi-amenaza & Centralizado & $\begin{array}{c}\text { http://190.60.210.210:8080/DGR/inde } \\
\text { x.jsf }\end{array}$ \\
\hline OSSO & Nacional & Tsunamis & Centralizado & http://www.osso.org.co/tsunami/ \\
\hline
\end{tabular}

A pesar de la tecnología que puedan utilizar, los SAT no están exentos de presentar fallas que comprometan su capacidad para difundir oportunamente una alerta, de ahí la importancia de proveer a las personas del conocimiento acerca de los fenómenos que pueden afectarlos; llegando ésta a ser la fuente primaria o única para detectar el peligro y actuar oportunamente (Hall, 2007). Un ejemplo claro es el caso de Tilly, la niña británica que en 2004 reconoció los indicios de los tsunamis gracias una lección de geografía en su colegio, y al observar la rápida reducción de la marea, alertó a su familia y otras 100 personas; quienes tuvieron tiempo de evacuar antes del impacto de las olas en la costa de Phuket, Tailandia. (Sampedro, 2005); o el caso de doña Leonor García, al saber que cuando la "plataneras crujen" se viene la tierra, y logra dar aviso a su familia, antes que el barro y las piedras sepultaran su casa durante la ola invernal de 2010-11 (El País, 2011).

\section{- Preparación y respuesta}

La población debe estar preparada para actuar ante una situación de riesgo, por tanto requiere el desarrollo de capacidades que le permitan identificar las amenazas a las que se encuentra expuesta, sus causas y consecuencias. Al respecto, los programas de educación son esenciales; así lo identificó la secretaría de la EIRD, en 2006, al lanzar la campaña "La prevención de los desastres empieza en la escuela", con el fin de sensibilizar gobiernos, comunidades e individuos para integrar estos temas en los currículos escolares. Cada plan de respuesta varía de acuerdo con la amenaza; una comunidad asentada en zona de ladera es vulnerable a la ocurrencia de deslizamientos, mientras que otra asentada en las costas marinas lo es a tsunamis y oleajes. Estos factores hacen que el trabajo con cada comunidad sea diferente; de allí la importancia de contextualizar cualquier intervención que se lleve a cabo.

Adicionalmente, la preparación debe estar integrada a los demás elementos que conforman el SAT, porque de poco sirve tener un aviso temprano y acertado, si las personas no saben cómo actuar o no poseen un plan de emergencia con rutas de evacuación o posibles albergues. Aunque muchos técnicos reconocen que los desastres tienen sus raíces en las estructuras sociales, no consideran este componente en los trabajos e investigaciones; siendo esta una dimensión fundamental que no ha sido suficientemente desarrollada en la gestión de los SAT. Finalmente, al ser los SAT una herramienta enmarcada dentro de la gestión del riesgo, resulta 
infructuoso que al ocurrir un desastre, así el SAT haya funcionado adecuadamente, no existan los mecanismos para brindar soluciones a las personas afectadas, que en la mayoría de los casos pierden viviendas y medios de sustento.

Un ejemplo de los beneficios de un SAT desarrollado en conjunto con la comunidad, fortalecido e integrado adecuadamente con otros mecanismos de prevención, tales como estrictos códigos de construcción sismo-resistente y concientización pública; se comprobó en 2011, ante el tsunami que azotó la costa oriental de Japón, luego de uno de los terremotos de mayor magnitud en el último siglo. Gracias al SAT se evitó una tragedia mayor, porque tanto la detección como emisión de la alarma fueron oportunas, permitieron la activación de los planes de respuesta y el desplazamiento hacia terrenos elevados, con rutas de evacuación claramente señalizadas (PNUD, 2014).

\section{CONCLUSIONES Y RECOMENDACIONES}

Para que sea posible el desarrollo de comunidades resilientes, como parte de los compromisos adquiridos por Colombia en el MAH, es necesario el cambio de paradigmas institucionales, modificando las estructuras y normas, para promover mejoras en la construcción de resiliencia humana. Las políticas estatales y las redes de apoyo comunitario pueden y deben facultar a las personas para superar las amenazas, cuando y donde quiera que surjan. La implementación de los SAT constituye una herramienta capaz de ayudar a la consecución de este objetivo, no sólo con la idea de alertar, sino también de proporcionar capacidades a las personas.

El desarrollo de capacidades como una forma de lograr mejoras sostenidas y continuas, gana cada vez más espacio en la búsqueda por reducir la vulnerabilidad vinculada a los ciclos de vida de las personas. Esta situación lleva a replantear que la gestión del riesgo debe abordarse con un enfoque participativo, involucrando a las comunidades en los diferentes procesos y sus métodos tradicionales, pues es la comunidad quien conoce e identifica las necesidades de su entorno.

Retomar el conocimiento y experiencias ancestrales es una estrategia importante, especialmente en las culturas andinas, que han desarrollado sistemas para predecir el tiempo a través de la observación del comportamiento fenológico de ciertas plantas y conductas de animales; adaptándose de manera eficaz a los eventos meteorológicos a través del tiempo. Estas acciones de integración pueden facilitar la aceptación y asimilación, por parte de la comunidad, de cualquier proyecto que se quiera desarrollar en su territorio y a la vez complementarlo.

El fomento de los SAT y la generación de alianzas maximizan sinergias que contribuyen a la óptima gestión del conocimiento de los productos y servicios que ofrecen estos sistemas, y que al ser integrados, mejoran el entendimiento y la importancia que tienen en la gestión del riesgo. Una mirada integral de los cuatro componentes de un SAT permite conseguir un enfoque holístico de su gestión, contribuyendo eficazmente con la buena gobernanza, la preparación, adaptación, la mitigación y la prevención.

Aunque los SAT, y en general la gestión del riesgo, estén liderados por técnicos, y centren casi toda la atención en el fortalecimiento tecnológico; estos sistemas deben gestionarse con la articulación de todos los aspectos primarios necesarios en el 
proceso de alertar anticipadamente (Hall, 2007): población, medios de comunicación, científicos y servidores públicos.

En el país, varios de los SAT implementados en la actualidad, no se encuentran integrados entre sí; situación que genera desconocimiento y sectorización de la información, reduciendo su efectividad. El uso de páginas web para la difusión de la información, aunque es un medio de comunicación masiva, tiene reducida divulgación hacia la población rural, debido al restringido acceso a internet en zonas rurales; a esto se suma que cada sistema utiliza un diseño y enfoque particular que genera heterogeneidad en su difusión y limita su utilidad.

La articulación funcional entre los diferentes SAT con un mismo lenguaje, facilita la integración entre sistemas y el intercambio de información, resultados y experiencias, tanto en el país como fuera de éste. Esto es un reto para el país, ya que en la actualidad no existe una comunidad de SAT, sino muchas y muy variadas entre sí, que podrían multiplicar esfuerzos colectivos para alcanzar los compromisos adquiridos por Colombia en los diferentes acuerdos internacionales de reducción del riesgo.

La cooperación regional en Latinoamérica para hacer frente a fenómenos naturales, que trascienden las fronteras de los países de la región, afectándolos simultáneamente, como es el caso del ENOS, en sus fases extremas, debe ser fortalecida. Este trabajo cooperativo se observa en otros lugares del mundo como en el continente europeo, donde los SAT han sido integrados en un único sistema llamado "Meteoalar". En Asia y África sucede lo mismo con el Regional Integrated MultiHazard Early Warning System for Africa and Asia (RIMES), y en Centroamérica con el SAT para Centroamérica. El reconocimiento de las amenazas de gran escala requiere una acción colectiva, que genere compromisos globales y promueva una mejor gobernanza internacional para enfrentarlas (PNUD, 2014).

\section{AGRADECIMIENTOS}

Al grupo de investigación IREHISA de la Universidad del Valle, y al Programa de Jóvenes Investigadores e Innovadores de COLCIENCIAS 2013, por su aporte al desarrollo de del proyecto "Diseño de un Sistema de Alerta Temprana Participativo para comunidades localizadas en la cuenca hidrográfica del río Tuluá".

\section{POTENCIAL CONFLICTO DE INTERESES}

Los autores declaran que no existe conflicto de interés en torno a la realización de la investigación. 


\section{FUENTE DE FINANCIACIÓN}

El presente artículo es resultado del proyecto "Diseño de un Sistema de Alerta Temprana Participativo (SATP) para comunidades localizadas en la cuenca hidrográfica del río Tuluá" desarrollado por el Grupo de Investigación IREHISA, entre 2014 y 2015, y financiado por COLCIENCIAS a través del Programa de Jóvenes Investigadores e Innovadores 2013.

\section{REFERENCIAS}

Acosta, M. (2013). Sistemas de Alerta Temprana (S.A.T) para la Reducción del Riesgo de Inundaciones Súbitas y Fenómenos Atmosféricos en el Área Metropolitana de Barranquilla [versión electrónica]. Recuperado de: Link

Agencia Suiza para el Desarrollo \& COSUDE (2010). Implementación de un sistema de alerta temprana para la cuenca del río Combeima, departamento del Tolima Colombia. Bogotá, Colombia.

Aristizábal, E., Gonzales, T., Montoya, J. D., Vélez, J. I., Martínez, H. \& Guerra, A. (2011). Análisis de umbrales empíricos de lluvia para el pronóstico de movimientos en masa en el Valle de Aburra, Colombia [Versión electrónica]. Revista EIA, (15), pp. 95111. Recuperado de: Link

Bedoya, M., Contreras, C. \& Ruiz, F. (2010). Alteraciones del régimen hidrológico y de la oferta hídrica por variabilidad y cambio climático. En: IDEAM (2010). Estudio nacional del agua. (Cap. 7) [En línea]. Recuperado de: Link

Brown, M. E. \& Funk, C. C. (2008). Climate - Food security under climate change [versión electrónica]. Science, 319(5863), pp. 580-581. Recuperado de: Link

Campo, P. A., \& Zafra, K. (2014). Sistema electrónico inalámbrico de alerta temprana y monitoreo del comportamiento del nivel de los ríos de bajo costo (tesis de pregrado). Universidad San Buenaventura, Cali, Colombia.

Campos, A., Holm, N., Díaz, C., Rubiano, D., Costa, C., Ramírez, F. \& Dickson, E. (2012).Resumen ejecutivo. Análisis de la gestión del riesgo de desastres en Colombia: un aporte para la construcción de políticas públicas. Banco Mundial. Bogotá. Recuperado de: Link

Carvajal, Y., Enciso, A., Rojas, D. \& Loaiza, W (2014). Isoyetas de precipitación máxima durante las crecientes históricas del río Cauca en su valle alto (1988 2011). Cali: Universidad del Valle; IREHISA; CVC-Corporación Autónoma Regional del Valle del Cauca.

Carvajal, Y. (2011). Inundaciones en Colombia ¿Estamos preparados para enfrentar la variabilidad y el cambio climático? [versión electrónica]. Revista Memorias, 9(16), pp. 105-119. Recuperado de: Link

Carvajal, Y., Correa, G. A. \& Muñoz, F. M. (2007). Modelos de predicción de caudal utilizando variables macroclimáticas y técnicas estadísticas multivariadas en el valle del río Cauca [Versión electrónica]. Ingeniería de Recursos Naturales y del Ambiente, (6), pp. 6-81. Recuperado de: Link 
Colombia Humanitaria. (2012). Ley 1523 de 2012: Sobre la primera política de gestión del riesgo de desastres en Colombia. Recuperado de: Link

Corpoguajira. (2011). Diseño de un sistema de alerta temprana por inundación y deslizamiento en el flanco nororiental de la Sierra Nevada de Santa Marta Departamento de La Guajira. Riohacha, Colombia. Recuperado de Link

Damman, G. 2008. Sistemas de información y alerta temprana para enfrentar el cambio climático: Propuesta de adaptación tecnológica en respuesta al cambio climático en Piura, Apurímac y Cajamarca. Perú, Lima: Soluciones Prácticas-ITDG. Recuperado de: Link

Domínguez, E. A., Angarita, H. \& Rivera, H. (2010). Viabilidad para pronósticos hidrológicos de niveles diarios, semanales y decadales en Colombia [versión electrónica]. Ingeniería e Investigación, 30(2), pp. 178-187. Recuperado de: Link

Domínguez, E. \& Lozano, S. (2014). Estado del arte de los sistemas de alerta temprana en Colombia [Versión electrónica]. Revista de la Academia Colombiana de Ciencias Exactas, Físicas y Naturales, 38(148), pp. 321-333. Recuperado de: Link

Estrategia Internacional para Reducción de Desastres - EIRD. (2006). La reducción de los desastres empieza en la escuela.

¿Qué tiene que hacer Cali para no hundirse en el invierno? (24 abril de 2011). El País. Recuperado de: Link

Echeverri, O. \& Valencia, Y. (2004). Análisis de los deslizamientos en la cuenca de la quebrada La Iguana de la ciudad de Medellín a partir de la integración lluvia-pendienteformación geológica. Dyna, 71(142), pp. 33-45. Recuperado de: Link

Fernández, C. \& Sabas, A. (2012). Sistema de Alerta Temprana centrado en la población para la cuenca media del río Otún [Versión electrónica]. Scientia et Technica, 2(50), pp. 211-217. Recuperado de: Link.

Fondo Adaptación - FONDO. (2012). Estudios previos para el rediseño, actualización y acompañamiento en la implementación y puesta en marcha del sistema de alertas tempranas de origen hidrometeorológico del IDEAM como apoyo técnico al sistema nacional para la gestión del riesgo de desastres de Colombia. Bogotá, Colombia.

García, M. C., Piñeros, A., Bernal, F. A. \& Ardila, E. (2012). Variabilidad climática, cambio climático y el recurso hídrico en Colombia [Versión electrónica]. Revista de Ingeniería, (36), pp. 60-64. Recuperado de: Link

Hall, P. (2007). Early warning systems: reframing the discussion [versión electrónica].Australian Journal of Emergency Management, 22(2), pp. 32-36. Recuperado de: Link

Grupo Intergubernamental de Expertos sobre el Cambio Climático - IPCC. (2012). Gestión de los riesgos de fenómenos meteorológicos extremos y desastres para mejorar la adaptación al cambio climático. Recuperado de: Link

Landa, R., Magaña, V. \& Neri, C. (2008). Agua y clima: elementos para la adaptación al cambio climático. SEMARNAT. Recuperado de: Link 
Lozano, O. (2011). Guía metodológica para incorporar la gestión del riesgo de desastres en la planificación de desarrollo. Lima, Perú: PREDES [versión electrónica]. Recuperado de: Link

Mantilla, G., Oliveros, H. \& Barnston, A. G. (2009). The role of ENSO in understanding changes in Colombia's annual malaria burden by region, 1960-2006 [versión electrónica].Malar J, 8(6), pp. 1-11. Recuperado de: Link

Montealegre, J.E. \& Pabón, J.D. (2000). La Variabilidad Climática Interanual asociada al ciclo El Niño-La Niña-Oscilación del Sur y su efecto en el patrón pluviométrico de Colombia [Versión electrónica]. Meteorología Colombiana, (2), pp. 7-21. Recuperado de: Link

Moreno, H., Vélez, M., Montoya, J. \& Rhenals, R. (2006). La lluvia y los deslizamientos de tierra en Antioquia: análisis de su ocurrencia en las escalas interanual, intraanual y diaria [Versión electrónica]. Revista EIA, 3(5), pp. 59-69. Recuperado de: Link

Narváez, L., Lavell, A. \& Pérez Ortega, G. (2009). La gestión del riesgo de desastres: un enfoque basado en procesos. Comunidad Andina. Secretaría General; Proyecto Apoyo a la Prevención de Desastres en la Comunidad Andina (PREDECAN). Recuperado de Link

National Oceanic and Atmospheric Administration - NOAA. (2012). Guía de referencia para sistemas de alerta temprana de crecidas repentinas. Recuperado de: Link

Ocharan, J. (2007). Sistemas de Alerta Temprana. Fotografía actual y retos futuros. Cuadernos Internacionales de Tecnología para el desarrollo humano. Recuperado de: Link

Organización de los Estados Americanos - OEA. (2010). Manual para el diseño, instalación, operación y mantenimiento de Sistemas Comunitarios de Alerta Temprana ante inundaciones. Washington: Organización de Estados Americanos. Recuperado de: Link

ONU \& EIRD (2008). El cambio climático y la reducción del riesgo de desastres. Nota informativa $N^{\circ}$ 1. Ginebra. Recuperado de: Link

Petrone, A. \& Petri, F. (2011). Umbrales hidrométricos de alerta: Río Coyolate, Escuintla (Guatemala). Convegno di Medio Termine dell' Associazione Italiana di Ingegneria Agraria. Belgirate: Universidad de Florencia. [En línea] Recuperado de: Link

Poveda, G. (2004). La hidroclimatología de Colombia: una síntesis desde la escala inter-decadal hasta la escala diurna [versión electrónica]. Revista Académica Colombiana Ciencias, 28(107), pp. 201-222. Recuperado de: Link

Poveda, G. \& Salazar, L. (2004). Annual and interannual (ENSO) variability of spatial scaling properties of a vegetation index (ndvi) in Amazonia [versión electrónica]. Remote Sensing of Environment 93(3), pp. 391-401. Recuperado de: Link

Programa de las Naciones Unidas para el Desarrollo - PNUD. (2014). Informe sobre desarrollo humano 2014. Sostener el progreso humano: Reducir vulnerabilidades y construir resiliencia. Recuperado de: Link 
Puertas, O. L., Carvajal, Y. \& Quintero, M. Q. (2011). Estudio de tendencias de la precipitación mensual en la cuenca alta-media del río Cauca, Colombia [versión electrónica]. Dyna, 78(169), pp. 112-120. Recuperado de: Link

Puertas, O. L. \& Carvajal, Y. (2008). Incidencia de El Niño-Oscilación del Sur en la precipitación y la temperatura del aire en Colombia, utilizando el Climate Explorer [versión electrónica]. Ingeniería \& Desarrollo, 23, pp. 114-118. Recuperado de: Link

Quispe, S. \& Aguilar, L.C. (2009). El Plan Comunal de Gestión del Riesgo Agrícola. Una herramienta de trabajo desde la experiencia del proyecto GRAC. Programa de Reducción del Riesgo de Desastres-PRRD 2007-2009. Alianza AGREPROCE, Fundación Agrecol Andes, PROSUKO, CEE.

Sampedro, J. (2005, 9 de enero). Cómo salvarse de un "tsunami". El País. Recuperado de: Link

Schuster, R. L. (1996). Socioeconomic significance of landslides. Landslides: Investigation and Mitigation. Washington (DC): National Academy Press. Transportation Research Board Special Report, 247, pp. 12-35.

Sánchez, R., Vargas, G., González, H. \& Pabón, D. (2001). Los fenómenos cálido del Pacífico (El Niño) y frío del Pacífico (La Niña) y su incidencia en la estabilidad de laderas en Colombia. En Colombia. III Simposio Panamericano de Deslizamientos ( $p$. 1-12). Cartagena: IDEAM. [En Línea] Recuperado de: Link

Sedano, K., Carvajal, Y. \& Díaz, A. (2013). Análisis de aspectos que incrementan el riesgo de inundaciones en Colombia [versión electrónica]. Luna Azul, (37), pp. 219238. Recuperado de: Link

Stern, N. (2007). El Informe Stern: La verdad sobre el cambio climático. Barcelona, España: Editorial Paidós Ibérica.

United Nations Educational, Scientific and Cultural Organization UNESCO. (2012).Conceptos y herramientas sobre sistemas de alerta temprana y gestión del riesgo para la comunidad educativa [En línea]. Recuperado de: Link

Unidad Nacional para la Gestión del Riesgo de Desastres - UNGRD. (s.f). Gobernanza para la gestión del riesgo. Recuperado de Link

Unidad Nacional Para la Gestión del Riesgo de Desastres - UNGRD. (2012). Líneas estratégicas y avances en priorización de zonas de intervención. Recuperado de: Link

United Nations International Strategy for Disaster Reduction UNISDR. (2009).Terminología sobre la reducción del riesgo de desastres. Recuperado de: Link

Villagrán, J., Scott, J., Cárdenas, C. \& Thompson, S. (2003). Sistemas de alerta temprana en el hemisferio americano. Contexto, estado actual y perspectivas futuras. Antigua, Guatemala.

Vincent, K. (2007). Uncertainty in adaptive capacity and the importance of scale [versión electrónica]. Global Environmental Change, 17(1), pp. 12-24. Recuperado de: Link 
1. Ingeniero Agrícola, Universidad del Valle. Joven Investigador de Colciencias, Grupo de Investigación en Ingeniería de Recursos Hídricos y Suelos -IREHISA-, Universidad del Valle, Colombia.juan.david.lopez.garcia@correounivalle.edu.co. orcid.org/00000002-2267-3957.

2. Ph.D. en Hidráulica y Medio Ambiente. Director Grupo de Investigación en Ingeniería de Recursos Hídricos y Suelos -IREHISA-, Universidad del Valle, Colombia.yesid.carvajal@correo.univalle.edu.co. orcid.org/0000-0002-2014-4226.

3. Ingeniera Agrícola, Universidad del Valle. Joven Investigador de Colciencias, Grupo de Investigación en Ingeniería de Recursos Hídricos y Suelos -IREHISA-, Universidad del Valle, Colombia. angelica.enciso@correounivalle.edu.co. orcid.org/0000-00018032-3130

4. Marco de Acción de Hyogo.

Para citar este artículo: López-García, J.D.; Carvajal-Escobar, Y y Enciso-Arango, A.M. (2017). Sistemas de alerta temprana con enfoque participativo: un desafío para la gestión del riesgo en Colombia. Revista Luna Azul, 44, 231-246. DOl: 10.17151/luaz.2017.44.14.

Recuperado de http://200.21.104.25/lunazul/index.php/component/content/article?id=230

Esta obra está bajo una Licencia de Creative Commons Reconocimiento CC BY (c) 